\title{
Zebrafish Kit ligands cooperate with erythropoietin to promote erythroid cell expansion
}

\author{
Jana Oltova ${ }^{1}$, Ondrej Svoboda ${ }^{1,2}$, Olga Machonova ${ }^{1}$, Petra Svatonova ${ }^{1}$, David Traver $^{2}$, Michal Kolar ${ }^{1}$, and \\ Petr Bartunek ${ }^{1}$ \\ ${ }^{1}$ Institute of Molecular Genetics of the Czech Academy of Sciences, Prague, Czech Republic \\ ${ }^{2}$ Department of Cellular and Molecular Medicine, University of California at San Diego, La Jolla, CA, USA.
}

Corresponding author:

Petr Bartunek

Institute of Molecular Genetics of the Czech Academy of Sciences

Videnska 1083, 14220 Prague 4, Czech Republic

+420296443117

bartunek@img.cas.cz

Short title (max 50 characters): Kitlg and Epo cooperation in erythropoiesis

Word count - main text: 4250

Word count - supplemental information: 800

Word count - abstract: 244

Figure/table count: 4 figures, 4 supplemental figures, 6 supplemental tables

\section{Reference count: 51}

\section{Key points:}

- Kit signaling contributes to erythroid cell development and is conserved from fish to man

- Ex vivo expansion and self-renewal of zebrafish erythroid progenitors requires addition of recombinant Kitlga 


\section{Abstract}

Kit ligand (Kitlg) is pleiotropic cytokine with a prominent role in vertebrate erythropoiesis. Although the role of Kitlg in this process has not yet been reported in Danio rerio (zebrafish), in the present study, we show that its function is evolutionary conserved. Zebrafish possess two copies of Kitlg genes (Kitlga and Kitlgb) due to whole genome duplication. To determine the role of each ligand in zebrafish, we performed a series of ex vivo and in vivo gain- and loss-of-function experiments. First, we tested the biological activity of recombinant Kitlg proteins in suspension culture from zebrafish whole kidney marrow and we demonstrate that Kitlga is necessary for expansion of erythroid progenitors ex vivo. To further address the role of kitlga and kitlgb in hematopoietic development in vivo, we performed gain-of-function experiments in zebrafish embryos, showing that both ligands cooperate with erythropoietin (Epo) to promote erythroid cell expansion. Finally, using the kita mutant ( $k i t a^{b 5 / b 5}$ or sparse), we show that Kita receptor is crucial for Kitlga/b cooperation with Epo in erythroid cells. In summary, using optimized suspension culture conditions with recombinant cytokines (Epo, Kitlga), we are reporting for the first time ex vivo suspension cultures of zebrafish hematopoietic progenitor cells, which can serve as an indispensable tool to study normal and aberrant hematopoiesis in zebrafish. Furthermore, we conclude that although partial functional diversification of Kit ligands has been described in other processes, in erythroid development, both paralogs play a similar role and their function is evolutionary conserved. 


\section{Introduction}

The most prominent cytokines that regulate proliferation and differentiation of erythroid cells in vertebrates are erythropoietin (EPO) $)^{1-3}$ and Kit ligand (KITLG or stem cell factor, SCF $)^{4-9}$. As was described in other vertebrates but not in zebrafish, binding of EPO and KITLG to their cognate receptors ensures erythroid lineage commitment by triggering specific signaling events ${ }^{1,8,10,11}$.

Besides its role in erythropoiesis, KITLG acts pleiotropically, affecting a wide range of tissues and cells, including hematopoietic (HSCs) and germ stem cells ${ }^{12-14}$. It is an important regulator that plays a role in many processes, both during ontogenesis and in the adult organism ${ }^{13,15}$. In mammals, KIT signaling is associated with erythro-myelopoiesis ${ }^{16}$, as well as neurogenesis and pigmentation ${ }^{13,17-19}$. Interestingly, there are two forms of KITLG occurring in vivo - transmembrane, important for the regulation of stem cells in their niches, and soluble, affecting more distant tissues ${ }^{13,20}$. Binding of KITLG to its receptor KIT, a member of receptor tyrosine kinase type-III family, leads to its autophosphorylation, further triggering various signaling cascades, including PI-3K, MAPK, SRC and JAK kinase pathways ${ }^{20,21}$.

In teleost species that include zebrafish, this scenario is more complex due to the fact that the whole ligand-receptor signalosome has been duplicated as a result of an extra round of whole genome duplication. Future studies are therefore dependent on the understanding of diversification of the functions of both ligands (Kitlga, Kitlgb) and receptors (Kita, Kitb) and their binding specificities. Similarly, there are also two copies of the epo gene present in the zebrafish genome - epoa and epob. The zebrafish epoa seems to play a similar role as its mammalian ortholog 22,23 but no role in hematopoiesis has been so far reported for epob (OS, $P B$, unpublished). For simplicity, we will use the designation Epo/epo for Epoa/epoa in this study.

Current data support the hypothesis that Kit receptor and Kit ligand paralogs have subspecialized during evolution. Kita is expressed in the neural crest, lateral line and notochord ${ }^{24}$. Overexpression of Kitlga results in hyper-pigmentation ${ }^{25}$ and kita receptor mutants ( kita $^{65 / 65}$ or sparse) have defective pigmentation ${ }^{24}$. On the other hand, the second zebrafish Kit paralog, Kitb, is expressed in neural tube and otic vesicles and likely does not play a role in melanogenesis ${ }^{26,27}$. Neither has a role in melanogenesis been reported for Kitlgb ${ }^{27}$.

Although studied extensively, the role of Kit signaling in zebrafish hematopoiesis has remained unknown for a long time. So far, there are only two studies that suggest potential roles of Kitlg in hematopoiesis in D. rerio. The first shows a mild increase of HSCs upon overexpression of $\mathrm{Kitlgb}^{28}$, the second reports a decrease in the number of HSCs upon downregulation of Kitb; however, no such phenotype was observed 
for Kita or Kitlga. Based on that, the authors concluded that neither Kita nor Kitlga are involved in hematopoiesis, but only in melanocyte formation, as suggested by previous studies ${ }^{27}$. Contradictory to the findings in other vertebrate models, and despite the fact that Kit receptors are expressed in hematopoietic tissues $^{24,27}$, hematopoiesis is not affected in the adult kita mutants ( $k i t a^{b 5 / b 5}$ or sparse $)^{24}$ under steady-state conditions.

The utilization of zebrafish as a model organism requires understanding of the regulatory mechanisms of hematopoietic lineage development and their evolutionary conservation, as well as proper approaches to study blood development and disease. The objective of this study is to better understand the importance of Kit ligands in zebrafish hematopoiesis, and thus providing important insights for further research of hematopoietic development. Although ex vivo expansion of erythroid progenitors has been reported in different vertebrate organisms ${ }^{29}$, a similar approach that would enable analysis of normal and aberrant hematopoiesis in zebrafish is not yet available. Here, we establish suspension culture conditions for the expansion of zebrafish erythroid progenitors and we investigate the role of the two zebrafish paralogs of Kit ligands (Kitlg) in zebrafish hematopoiesis using in vivo and ex vivo experimental approaches.

\section{Methods}

\section{Animal stocks and embryos}

Fish were mated, raised and staged according to "The zebrafish book. A guide for the laboratory use of zebrafish (Danio rerio)" ${ }^{\prime 30}$ and the recommendations for zebrafish husbandry and housing ${ }^{31}$. For tracking the animals (age, number, health, genotype), we used an in-house developed dedicated database solution called Zebrabase ${ }^{32}$. Fish were kept in ZebTEC aquatic system (Tecniplast). Transgenic reporter lines expressing fluorescent genes under the control of tissue specific promoters (gata1:DsRed ${ }^{33}$ and /cr:EGFP ${ }^{34}$, as well as mutant lines (kita ${ }^{\mathrm{b} 5 / \mathrm{b} 5}$ or sparse) ${ }^{24}$ and wild-type animals WT(AB) were used in this study. For ex vivo experiments, 6-month old fish were used to ensure optimal number and state of whole kidney marrow cells. Animal care and experiments were approved by the Animal Care Committee of the Institute of Molecular Genetics, Czech Academy of Sciences (13/2016 and 96/2018) in compliance with national and institutional guidelines.

\section{Ex vivo cultures}

Zebrafish whole kidney marrow cells were isolated as previously described ${ }^{35}$. Cells were fractionated using Biocoll $\left(1.077 \mathrm{~g} / \mathrm{ml}\right.$, Merck L6115) density centrifugation and initially seeded at $3 \times 10^{6} \mathrm{cells} / \mathrm{ml}$ and 
cultivated in zfS13 medium (for composition, see Supplemental Table 3 ) at $32^{\circ} \mathrm{C}$ and $5 \% \mathrm{CO}_{2}$. Carp serum was obtained by the procedure described and published previously ${ }^{35}$. Specific cytokines (Epo, Gcsfa, Kitlga and Kitlgb) were added at final concentrations of $(100 \mathrm{ng} / \mathrm{ml})$, dexamethasone (Dex) was added at final $1 \mu \mathrm{M}$. Cells were counted using CASY Cell Counter \& Analyzer (OMNI Life Science). Following days, cells were maintained at $2 \times 10^{6}$ cells $/ \mathrm{ml}$ and one third of the medium containing fresh cytokines and Dex was exchanged every other day to ensure optimal growth of the cells. To study the clonogenic potential of the whole kidney marrow cells after addition of different cytokines, we performed clonal assays in semisolid media (methylcellulose), which prevents movement of the single cells plated in it. For the detailed protocol for these assays, please refer to Svoboda et al., $2016^{35}$.

\section{Cytokine cloning and expression}

First, the amino acid sequence of zebrafish Kitlga/b, was subjected to protein structure prediction and hydrophobicity analysis using Phobius tool (http://phobius.sbc.su.se/). Two large hydrophobic regions (amino acid positions 1 to 24 and 206 to 224 for Kitlga, and amino acid positions 1 to 31 and 185 to 209 for Kitlgb) corresponding to the putative signal peptide (SP) and transmembrane (TM) domains, respectively, were identified (Supplemental Figure 1A). To generate a Kitlga/b version devoid of both domains, sequence specific primers (Supplemental Table 1) were used for PCR amplification of each Kitlg cDNA fragment corresponding to amino acid 25 to 182 (Kitlga) and 31 to 187 (Kitlgb) from adult zebrafish retina. We used the baculovirus expression system to produce soluble Kitlga/b in large quantities. We cloned the amplified fragment into modified PAc-GP67-B vector containing 6xHis and generated the recombinant baculovirus by co-transfection of pAc-His-Kitlga/b and BaculoGold Bright Baculovirus DNA into sf21 insect cells. Virus-infected cells expressed GFP (Supplemental Figure 1B) and secrete recombinant His-Kitlga/b extracellularly. Finally, we purified the secreted proteins on a Ni2+-NTA agarose column and used them for the following experiments (Supplemental Figure $1 \mathrm{C}$ ). To generate Kitlga/b the construct for mRNA injection experiments, the full length cDNA fragment was amplified using RT-PCR using sequence specific primers (Supplemental Table 1). Erythropoietin protein and mRNA has been prepared as previously described $22,35,36$.

\section{Additional methods}

For experimental details on the generation of recombinant cytokines, mRNA microinjection, benzidine staining, image analysis, qPCR, FACS-sorting, RNAseq and transcriptomics, please refer to supplemental methods. 


\section{Data sharing statement}

RNAseq data are available in ArrayExpress under accession number E-MTAB-8800. Plasmids for cytokine expression are available via Addgene with the accession numbers 140292 (pAc-His-zfKitlga) and 140293 (pAc-His-zfKitlgb).

\section{Results}

\section{Kit ligands promote erythroid and myeloid expansion of whole kidney marrow cells.}

First, we cloned and expressed recombinant zebrafish Kitlga and Kitlgb. We amplified the mature form of zebrafish kitlga and kitlgb lacking the signal peptide-encoding region, intracellular and transmembrane region (Supplemental Figure 1A) from adult retina and we produced recombinant Kitlga and Kitlgb in sf21 insect cells (Supplemental Figure 1B, C). His-tagged purified proteins were used in the following experiments.

To test the biological activity of Kitlg proteins, we designed an experiment using whole kidney marrow cells isolated from adult, 6-month old zebrafish. To reveal differences that would suggest biological activity and potential cooperation of Kitlg proteins with other recombinant cytokines, we treated the cells with various factors, or their combination, and subsequently counted them at specific time points. After 3 days in culture, the potential enhancement in the myeloid lineage was assessed. We added both Kit ligands, either separately or combined with Gcsfa, which has been previously shown to support myeloid cell fate ${ }^{37}$. Interestingly, we observed an increase in the number of cells in all the conditions tested when compared to untreated control (Figure 1A). In agreement with previously published studies, Gcsfa promoted significantly the growth of whole kidney marrow cells, and the effect was further potentiated by the addition of either of the Kit ligands. This additional effect tended to be similar for both Kit ligand paralogs, however this cooperation proved to be statistically significant only for Kitlgb, but not Kitlga. The Kit ligands alone exhibited reproducible mild activity that was, however, statistically not significant. To verify that the addition of Gcsfa along with Kitlga or Kitlgb promoted myelopoiesis, we morphologically characterized cultured cells (Supplemental Figure 2A, top). At day 3, the culture mostly comprised of monocytes and macrophages. Although the addition of Kitlga or Kitlgb to Gcsfa increases cumulative number of cells (as shown in Figure 1A), the overall composition of cell culture and proportion of different cell types remained similar with or without the Kit ligands. 
Next, we tested the activity of Kit ligands during erythroid cell expansion. Cells were treated with both Kitlga and Kitlgb, either separately or combined with Epo. The increase in cell number was quantified after 7 days in culture. In agreement with previously published studies ${ }^{22,36}$, Epo boosted cell growth significantly and again, there was a mild increase in the number of the cells when treated with any of the Kit ligands alone. Strikingly, when Epo was combined with Kitlga, we measured a significant increase in the number of cells at 7 days in culture compared to Epo alone (Figure 1B). Although, the additive effect of Kitlgb was not significant, the trend to increase the number of cells in the presence of Epo was still observed. To examine composition of cell culture, we further performed morphological characterization of cultured cells. At day 7, the culture mostly comprised of erythroid cells upon Epo stimulation as expected (Supplemental Figure 2A, bottom) and the addition of Kitlga led to an increase in number of erythroid progenitors, which explains elevated number of cells in this culture.

With the aim of maximum expansion of erythroid progenitors, we decided to improve the conditions for suspension culture of whole kidney marrow cells. Although there were no existing reports of suspension culture of zebrafish or hematopoietic progenitor cells, we supposed that similar conditions might be effective in suspension cultures as was shown in human and mouse ${ }^{10,38}$. As different steroids were reported as important players in the maintenance of self-renewal and proliferation of erythroid progenitors in chicken, mouse and human ${ }^{38-41}$, we included Dex and tested its different concentrations and combinations together with Epo and Kitlga, the more potent Kitlg paralogue.

We found that, in line with previous findings in other vertebrates, Kitlga, Epo and Dex act synergistically in ex vivo cultures, enabling erythroid expansion (Figure $1 C$, left). When compared to the cells treated with Epo and Dex, cells treated with Epo, Dex and Kitlga formed islets of round, highly gata1:DsRed-positive cells, corresponding to erythroid progenitors (Figure 1C, right). Additionally, we observed that Epo can cooperate with Kitlga to promote the growth of large erythroid colonies in colony forming assays in methylcellulose (Supplemental Figure 2B). When Kitlga was combined with Epo in kidney marrow culture from gata1:DsRed transgenic fish, we observed significantly more, highly gata1:DsRed positive colonies that were also significantly larger (Supplemental Figure 2C, D).

Kitlga treatment, when combined with Epo and dexamethasone, leads to upregulation of erythroid-, translation- and cell cycle-related gene expression

To gain deeper insight into the mechanism of Kitlga action when combined with Epo and Dex in kidney marrow suspension cultures, we performed an RNAseq experiment, comparing samples from Epo, Dex, Kitlga and Epo, Dex-treated cells. Samples were collected after 8 days in culture. This was the first 
time-point where we could observe a significant increase in proliferation of erythroid cells in the presence of Epo, Dex and Kitlga, as shown in the growth curve (Figure 1C, left).

RNAseq analysis revealed that both culture conditions resulted in high expression of erythroid marker genes (data not shown). However, addition of Kitlga to the Epo, Dex combination caused an additional increase in expression of erythroid genes. Among the upregulated differentially expressed genes (see heatmap in Figure 1D and Supplemental Table 4), we identified several erythroid (g6fl, lias) and cell cycle ( $c d c 27)$ specific genes, confirming additional Kitlga-mediated erythroid cell expansion over Epo and Dex treatment alone. On the contrary, downregulated genes were mostly linked to the myeloid cell lineage (pigr/2.3, blnk, nfkbiz, alox5a, inpp5d), providing further evidence that Epo, Dex, Kitlga-induced expansion led to a more enriched erythroid cell population as compared to Epo, Dex culture alone. Next, we examined the expression of both kit receptor and revealed that the expression of kita is predominant in adult erythroid progenitor cells (average raw counts 285 (kita) vs. 3 (kitb)).

Finally, we were interested specifically in changes in expression of erythroid-specific genes. The analysis of RNAseq data reveled that, compared to cells treated with Epo and Dex, cells treated with Epo, Dex and Kitlga display higher expression of many of the important erythroid genes - namely globins (hbae1.1, hbaa1), genes involved in heme and iron metabolism (e.g. urod, slc11a2, steap3), erythroid cytoskeleton (e.g. rhd, sptb, eph41b) and signaling (gata1a, jak3) (Figure 1E, Supplemental Table 5). Based on GO prediction, we revealed an enrichment in specific categories of biological processes - namely in translation (GO:0006412, GO:0002181, GO:0000028, GO:0000027), erythropoiesis (GO:0042541, GO:0030218, GO:0048821) and cell cycle regulation (GO:0051726). For a full list of enriched GO terms in all three standard GO term categories, refer to Supplemental Table 6.

\section{Both Kit ligands cooperate with Epo to promote erythroid expansion in zebrafish embryos}

To test the function of both Kit ligands in vivo, we microinjected cytokine mRNA(s) into 1-cell stage embryos. When combined with epo, both kitlga and kitlgb showed an enhancement in /cr:EGFP expression (Figure 2A) and hemoglobinization (Figure 2D) at $72 \mathrm{hpf}$ compared to epo alone. Quantification of Icr:EGFP positive cells in the tail region (Figure 2B), FACS analysis of total Icr:EGFP positive cells (Figure $2 \mathrm{C}$ and Supplemental Figure 3) as well as benzidine staining in the tails (Figure 2E) of $72 \mathrm{hpf}$ embryos confirmed the observed trend. Note that even though similar trend to increase number of erythroid cells, in combinations with epo, was observed for both Kit ligands under all three experimental readouts, the effect was more robust and statistically significant only for kitlgb in all types of experiments. Next, quantitative PCR (qPCR) analysis from whole injected embryos also showed an increase in the expression of erythroid 
markers at $72 \mathrm{hpf}$ (Figure 2F) when epo was combined with either of the ligands, however, these changes were rather subtle. Finally, in agreement with previous studies, we observed increased number of melanocytes after the overexpression of Kitlga but not Kitlgb.

\section{The expression of both kit receptors is gradually increasing during embryonic development}

To better understand the mechanism of cooperation of Epo with Kit ligands, we first analyzed the expression of both kit receptors in tissues and during the development by qPCR. Among all the tissues analyzed, the highest kita expression was observed in kidney (Supplemental Figure 4A) while the highest kitb expression was observed in the retina (Supplemental Figure 4B). During zebrafish embryonic development, the highest relative expression was observed at $7 \mathrm{dpf}$ (kita) and $72 \mathrm{hpf}$ (kitb) (Figure 3A). As we saw a significant expansion of erythroid, gata1:DsRed+cells in ex vivo cultures, we aimed to analyze the dynamics of the receptor expression in erythroid cells during development. Therefore, we collected samples from $24 \mathrm{hpf}, 48 \mathrm{hpf}$ and $72 \mathrm{hpf}$ gata1:DsRed and /cr:EGFP-embryos and FACS-sorted gata1:DsRedpositive cells (Figure 3B) or Icr:EGFP-positive cells (Figure 3C), respectively. In both cases, we found that there is a gradually increasing expression of both receptors in erythroid cells during the course of early development.

\section{Kita mediates Kitlg-dependent erythroid expansion in vivo}

To understand the molecular basis of the effect we observed on erythroid cells, we used the sparse mutant $\left(k_{i t a}{ }^{b 5 / b 5}\right)$ zebrafish, lacking the functional Kita receptor. We injected mRNA for epo, kitlga and kitlgb. We observed that in sparse mutants, the number of erythrocytes is slightly decreased in epo injected embryos and the effect of cooperation between any of the kitlg and epo is completely lost (Figure 4A). These findings are also supported by qPCR data showing that the expression of $\beta$-globin (hbbe1) and gata1a is not increased after the injection of epo with either of the kit ligands, as is the case in wild-type embryos (Figure 4B).

To summarize, we have cloned and expressed both Kit ligand zebrafish paralogs. Using the purified recombinant proteins, we tested various cytokine combinations in suspension culture of whole kidney marrow cells and defined an optimal factor combination, composed of Epo, Kitlga and dexamethasone, which enables efficient expansion of erythroid progenitors. Also, we tested gain of function effect of both kitlga and kitlgb in embryonic hematopoiesis and observed an increase in the number of erythroid cells in epo, kitlgb and epo, kitlgb mRNA-injected fish compared to epo alone. Finally, by employing the kita (sparse) mutants, we showed that Kita is mediating Kitlg-dependent erythroid expansion in vivo. 


\section{Discussion}

In this study, we explored the role of zebrafish Kit ligands in hematopoiesis. In zebrafish, multiple paralogs of many important genes resulted from an extra round of whole genome duplication 250-350 million years ago $^{42,43}$, including hematopoietic cytokines. After such an event, the process of pseudogenization often leads to a loss of function of one of the paralogs ${ }^{44-46}$. Alternatively, paralogs of an ancestral gene can either remain functionally redundant, split the original function between the two (subfunctionalization), or acquire completely new functions (neofunctionalization) ${ }^{47}$. Here, we studied the hematopoietic function of two retained zebrafish paralogs of a crucial hematopoietic cytokine, the Kit ligand (KITL or Stem Cell Factor - SCF), designated as Kitlga and Kitlgb.

So far, KIT signaling has not been associated with erythropoiesis in zebrafish ${ }^{24,25}$. This is in contrast with previous studies in human ${ }^{8}$, mouse ${ }^{4}$ and chicken $^{9,16,48}$, where it has been shown that KITL is critical for proper erythroid development ${ }^{5,15,49}$. By focusing on Kit function during erythropoiesis in zebrafish, we were able to prove for the first time, using ex vivo and in vivo gain- and loss-of-function experiments, that Kit signaling does indeed play a role in zebrafish erythroid differentiation. Based on RNAseq and GO analysis, this likely happens in a similar manner as in other vertebrate species via induction of cell proliferation coupled with differentiation ${ }^{40,48}$, promoting cell cycle progression and enhancing protein translation in differentiating cells.

To examine the role of Kit ligands ex vivo, we used clonal assays and liquid culture experiments 23,36,37,50. Since the reported cross-reactivity of mammalian hematopoietic cytokines in zebrafish is very limited ${ }^{35}$, it was essential to use bona fide zebrafish Kit ligands as recombinant proteins. Similarly as described for other cytokines ${ }^{7,9,35,36}$, we cloned the extracellular portion of the corresponding cDNAs, lacking the $\mathrm{N}$-terminal signal peptide, transmembrane domain and cytosolic part of both cytokines. This approach allowed us to generate soluble form of Kit ligands to stimulate kidney marrow progenitor cells ex vivo ${ }^{8,13}$.

As a result, treatment of hematopoietic progenitor cells isolated from kidney marrow with Kitlga, in combination with the other master regulator of erythroid lineage commitment, Epo ${ }^{22,23}$, enabled erythroid expansion of cells in suspension cultures and clonal assays, as in other vertebrates ${ }^{1,8,10,11}$. Such an effect was observed also for Kitlgb, however its cooperation with Epo was less pronounced and was not statistically significant. We hypothesize that soluble form of Kitlgb that lacks the transmembrane and cytosolic domain is less effective to stimulate differentiation of hematopoietic progenitors towards 
erythroid lineage and that the main role of Kitlgb is stimulation of neighboring cells in stem cell niches via contact dependent signaling.

Next, we also tested the activity of Dex in addition to Epo and Kitlga, the more effective soluble Kitlg paralogue. Dex is a glucocorticoid receptor agonist that has been reported to support self-renewal and differentiation of erythroid progenitors ${ }^{10,38-40}$ and indeed, addition of Dex to Kitlga and Epo led to synergistic erythroid expansion in suspension cultures.

To understand the mechanism triggering this substantial cell expansion, we performed an RNAseq experiment. We decided to compare the effect of Epo and Dex versus Epo, Dex, and Kitlga treatment on adult kidney marrow cells. Transcriptional profiling showed expression of erythroid specific genes in both conditions, as expected due to Epo-mediated signaling. However, we observed relatively subtle changes in expression between the Epo and Dex and the Epo, Dex, and Kitlga cultures. The trends of expression changes showed a distinct erythroid fingerprint in cells treated with Epo, Dex and Kitlga, compared to a more mixed/myeloid one in cells treated with Epo and Dex alone, as suggested by the increased expression of myeloid-specific genes. In addition, we found that the prominent receptor expressed in adult erythroid progenitor cells is kita, indicating that the effect of erythroid expansion is mediated by this receptor. This corresponds to the fact that isolation of kidney marrow cells yields, in addition to progenitor cells, also a fraction of myeloid cells with the ability to survive in the culture. However, this effect becomes negligible during erythroid expansion of cells treated with Epo, Dex, and Kitlga, when myeloid cells are overgrown by expanding erythroid progenitors. Importantly, gene ontology analysis revealed an enrichment especially in translation and expression of erythroid-specific genes in Epo, Dex and Kitlga cultures. Therefore, we hypothesize that Kitlga might enhance erythroid cell development and cell proliferation potentially through upregulation of genes related to protein translation and cell cycle progression.

To confirm these findings, we decided to test, whether Kit signaling is involved also in zebrafish erythropoiesis in vivo using full length form of both kit ligands. For ex vivo experiments, we used soluble forms, whereas for in vivo experiments, we used the full-length coding sequence, including transmembrane and cytosolic domains, as used in previous reports ${ }^{27}$. It has been shown that the function of soluble and transmembrane forms under physiological conditions is different ${ }^{13}$. The soluble form of the protein is distributed throughout the whole organism via circulation and affect distant tissues. It has been shown that this form is required and sufficient for proper erythroid signaling in cultured cells ${ }^{16}$. On the other hand, according to previous studies, the major site of action of the transmembrane form of Kit ligand might be in stem cell niches ${ }^{13}$. We hypothesize that full length Kitlgb can contribute, in addition to HSPCs ${ }^{27}$, 
also to erythroid expansion in vivo. As a result, we found that the full length kitlga was potent in expansion of erythroid cells at $72 \mathrm{hpf}$ when injected together with epo mRNA. Interestingly, similarly to kitlga, also the kitlgb was able to mediate such erythroid expansion, when injected in vivo in full-length form, but this effect was markedly decreased in ex vivo experiments using short soluble form. This supports our hypothesis that there is a difference in potency between soluble and full-length form of Kitlgb. To prove this, we generated shortened kitlga and kitlgb mRNA, corresponding to the soluble region of Kitlg proteins and indeed, there was a decrease in a potency of soluble kitlgb to stimulate erythropoiesis (data not shown), whereas soluble kitlga remained equally active.

Finally, we investigated, if Kita receptor is dispensable in Kitlg-mediated erythroid cell expansion in vivo. As a result, we did not observe the cooperation of epo with either of the kit ligands in erythroid differentiation in kita (sparse) mutant fish. These data suggest that Kita is responsible for Kit signal transduction in erythroid cells. Although Kitb might also be involved in this process, it couldn't compensate for missing Kita, and Kita seems to be required for Kit signaling in zebrafish erythroid cells. In addition to this, we also observed decreased number of erythroid cells in both, control and in epo injected, sparse mutants when compared to the wild-type embryos. This indicates that endogenous Kit signaling plays role also under physiological conditions during the normal erythropoiesis.

To summarize, in this study we have addressed the question of the role of Kitlg in hematopoiesis in zebrafish using both in vivo and ex vivo approaches. Importantly, we established optimized liquid culture conditions for erythroid expansion of kidney marrow progenitor cells that require the addition of Kitlga. Together with colony forming assays, suspension cultures might serve as indispensable tools in disease modelling, allowing analysis of normal and aberrant hematopoiesis in zebrafish blood mutants (vlade, moonshine, mindbomb etc. $)^{51}$. This will enable further biochemical, genomic and proteomic analysis of ex vivo cultured and expanded cells.

Interestingly, zebrafish Kitlga and Kitlgb seem to possess only partial redundancy in its function: Kitlga retains the role in melanogenesis and pigmentation, which is lost in the case of Kitlgb, but both ligands potentially contribute to the overall process of erythroid commitment and development in zebrafish in vivo. Although this function is not required in steady state erythroid development, it might become important in stress conditions. The exact mechanism of functional diversification of Kit ligands has yet to be investigated but altogether, we demonstrate that role of Kit signaling is evolutionarily conserved with respect to erythroid development as previously unnoticed ${ }^{24}$, and therefore it strengthens the use of zebrafish as a model to study normal and aberrant human hematopoiesis. 


\section{Acknowledgements}

We thank Nikol Pavlu,Tereza Hojerova and Tereza Hingarova for animal care, Tereza Mikulasova and Martina Hason, for graphical work and cDNA samples, Trevor Epp for editing the manuscript and Leonard Zon for providing gata1:DsRed and /cr:EGFP reporter fish lines. Supported by the Czech Science Foundation (16-21024S), Ministry of Health (NV19-07-00412) and 68378050-KAV-NPUI to PB. OS was partially funded by American Heart Association (19POST34380328).

\section{Authorship contribution}

J.O., O.M., O.S. performed the experiments, J.O, O.S., P.S., D.T., M.K., and P.B. designed the experiments, analyzed the data and wrote the manuscript.

\section{Conflict of interest}

The authors declare no conflict of interest. 


\section{References}

$1 \quad$ Krantz, S. B. Erythropoietin. Blood 77, 419-434 (1991).

2 Goldwasser, E., Krantz, S. B. \& Wang, F. F. Erythropoietin and erythroid differentiation. Symposium on Fundamental Cancer Research 37, 103-107 (1984).

3 Graber, S. E. \& Krantz, S. B. Erythropoietin and the control of red cell production. Annual review of medicine 29, 51-66, doi:10.1146/annurev.me.29.020178.000411 (1978).

4 Antonchuk, J., Hyland, C. D., Hilton, D. J. \& Alexander, W. S. Synergistic effects on erythropoiesis, thrombopoiesis, and stem cell competitiveness in mice deficient in thrombopoietin and steel factor receptors. Blood 104, 1306-1313, doi:10.1182/blood-2004-04-1522 (2004).

5 Huang, E. et al. The hematopoietic growth factor KL is encoded by the SI locus and is the ligand of the c-kit receptor, the gene product of the W locus. Cell 63, 225-233, doi:10.1016/0092-8674(90)90303-v (1990).

6 Toksoz, D. et al. Support of human hematopoiesis in long-term bone marrow cultures by murine stromal cells selectively expressing the membrane-bound and secreted forms of the human homolog of the steel gene product, stem cell factor. Proceedings of the National Academy of Sciences of the United States of America 89, 7350-7354 (1992).

7 Andrews, R. G. et al. Recombinant human stem cell factor, a c-kit ligand, stimulates hematopoiesis in primates. Blood 78, 1975-1980 (1991).

8 Broudy, V. C. Stem cell factor and hematopoiesis. Blood 90, 1345-1364 (1997).

9 Bartunek, P. et al. Avian stem cell factor (SCF): production and characterization of the recombinant His-tagged SCF of chicken and its neutralizing antibody. Cytokine 8, 14-20, doi:10.1006/cyto.1996.0003 (1996).

10 Panzenbock, B., Bartunek, P., Mapara, M. Y. \& Zenke, M. Growth and differentiation of human stem cell factor/erythropoietin-dependent erythroid progenitor cells in vitro. Blood 92, 3658-3668 (1998).

11 Katakura, F. et al. Exploring erythropoiesis of common carp (Cyprinus carpio) using an in vitro colony assay in the presence of recombinant carp kit ligand $\mathrm{A}$ and erythropoietin. Developmental and comparative immunology 53, 13-22, doi:10.1016/j.dci.2015.06.006 (2015). 
12 Bokemeyer, C. et al. Expression of stem-cell factor and its receptor c-kit protein in normal testicular tissue and malignant germ-cell tumours. J Cancer Res Clin Oncol 122, 301-306, doi:10.1007/bf01261407 (1996).

13 Lennartsson, J. \& Ronnstrand, L. Stem cell factor receptor/c-Kit: from basic science to clinical implications. Physiol Rev 92, 1619-1649, doi:10.1152/physrev.00046.2011 (2012).

14 Pesce, M., Di Carlo, A. \& De Felici, M. The c-kit receptor is involved in the adhesion of mouse primordial germ cells to somatic cells in culture. Mechanisms of development 68, 37-44, doi:10.1016/s0925-4773(97)00120-2 (1997).

15 Ding, L., Saunders, T. L., Enikolopov, G. \& Morrison, S. J. Endothelial and perivascular cells maintain haematopoietic stem cells. Nature 481, 457-462, doi:10.1038/nature10783 (2012).

16 Hayman, M. J., Meyer, S., Martin, F., Steinlein, P. \& Beug, H. Self-renewal and differentiation of normal avian erythroid progenitor cells: regulatory roles of the TGF alpha/c-ErbB and SCF/c-kit receptors. Cell 74, 157-169 (1993).

17 Grabbe, J., Welker, P., Dippel, E. \& Czarnetzki, B. M. Stem cell factor, a novel cutaneous growth factor for mast cells and melanocytes. Arch Dermatol Res 287, 78-84, doi:10.1007/bf00370723 (1994).

18 Alexeev, V. \& Yoon, K. Distinctive role of the cKit receptor tyrosine kinase signaling in mammalian melanocytes. J Invest Dermatol 126, 1102-1110, doi:10.1038/sj.jid.5700125 (2006).

19 Gong, Y. et al. SCF/SCFR signaling plays an important role in the early morphogenesis and neurogenesis of human embryonic neural retina. Development 146, doi:10.1242/dev.174409 (2019).

20 Ashman, L. K. The biology of stem cell factor and its receptor C-kit. The international journal of biochemistry \& cell biology 31, 1037-1051, doi:10.1016/s1357-2725(99)00076-x (1999).

21 Abbaspour Babaei, M., Kamalidehghan, B., Saleem, M., Huri, H. Z. \& Ahmadipour, F. Receptor tyrosine kinase (c-Kit) inhibitors: a potential therapeutic target in cancer cells. Drug Des Devel Ther 10, 2443-2459, doi:10.2147/DDDT.S89114 (2016).

22 Paffett-Lugassy, N. et al. Functional conservation of erythropoietin signaling in zebrafish. Blood 110, 2718-2726, doi:10.1182/blood-2006-04-016535 (2007).

23 Stachura, D. L. et al. Clonal analysis of hematopoietic progenitor cells in the zebrafish. Blood 118, 1274-1282, doi:10.1182/blood-2011-01-331199 (2011). 
24 Parichy, D. M., Rawls, J. F., Pratt, S. J., Whitfield, T. T. \& Johnson, S. L. Zebrafish sparse corresponds to an orthologue of c-kit and is required for the morphogenesis of a subpopulation of melanocytes, but is not essential for hematopoiesis or primordial germ cell development. Development 126, 3425-3436 (1999).

25 Hultman, K. A., Bahary, N., Zon, L. I. \& Johnson, S. L. Gene Duplication of the zebrafish kit ligand and partitioning of melanocyte development functions to kit ligand a. PLoS genetics 3, e17, doi:10.1371/journal.pgen.0030017 (2007).

26 Mellgren, E. M. \& Johnson, S. L. kitb, a second zebrafish ortholog of mouse Kit. Development Genes and Evolution 215, 470-477, doi:10.1007/s00427-005-0001-3 (2005).

27 Mahony, C. B., Pasche, C. \& Bertrand, J. Y. Oncostatin M and Kit-Ligand Control Hematopoietic Stem Cell Fate during Zebrafish Embryogenesis. Stem cell reports 10, 1920-1934, doi:10.1016/j.stemcr.2018.04.016 (2018).

28 Mahony, C. B., Fish, R. J., Pasche, C. \& Bertrand, J. Y. tfec controls the hematopoietic stem cell vascular niche during zebrafish embryogenesis. Blood 128, 1336-1345, doi:10.1182/blood-2016-04710137 (2016).

29 von Lindern, M. et al. The glucocorticoid receptor cooperates with the erythropoietin receptor and c-Kit to enhance and sustain proliferation of erythroid progenitors in vitro. Blood 94, 550-559 (1999).

30 Westerfield, M. A guide for the laboratory use of zebrafish (Danio rerio). 4th ed., (Univ. of Oregon Press, Eugene, 2000).

31 Alestrom, P. et al. Zebrafish: Housing and husbandry recommendations. Lab Anim, 23677219869037, doi:10.1177/0023677219869037 (2019).

32 Oltova, J. et al. Zebrabase: An Intuitive Tracking Solution for Aquatic Model Organisms. Zebrafish 15, 642-647, doi:10.1089/zeb.2018.1609 (2018).

33 Traver, D. et al. Transplantation and in vivo imaging of multilineage engraftment in zebrafish bloodless mutants. Nature immunology 4, 1238-1246, doi:10.1038/ni1007 (2003).

34 Ganis, J. J. et al. Zebrafish globin switching occurs in two developmental stages and is controlled by the LCR. Developmental biology 366, 185-194, doi:10.1016/j.ydbio.2012.03.021 (2012). 
35 Svoboda, O. et al. Ex vivo tools for the clonal analysis of zebrafish hematopoiesis. Nature protocols 11, 1007-1020, doi:10.1038/nprot.2016.053 (2016).

36 Stachura, D. L. et al. Zebrafish kidney stromal cell lines support multilineage hematopoiesis. Blood 114, 279-289, doi:10.1182/blood-2009-02-203638 (2009).

37 Stachura, D. L. et al. The zebrafish granulocyte colony-stimulating factors (Gcsfs): 2 paralogous cytokines and their roles in hematopoietic development and maintenance. Blood 122, 3918-3928, doi:10.1182/blood-2012-12-475392 (2013).

38 Kolbus, A. et al. Cooperative signaling between cytokine receptors and the glucocorticoid receptor in the expansion of erythroid progenitors: molecular analysis by expression profiling. Blood 102, 31363146, doi:10.1182/blood-2003-03-0923 (2003).

39 England, S. J., McGrath, K. E., Frame, J. M. \& Palis, J. Immature erythroblasts with extensive ex vivo self-renewal capacity emerge from the early mammalian fetus. Blood 117, 2708-2717, doi:10.1182/blood2010-07-299743 (2011).

40 Leberbauer, C. et al. Different steroids co-regulate long-term expansion versus terminal differentiation in primary human erythroid progenitors. Blood 105, 85-94, doi:10.1182/blood-2004-031002 (2005).

41 Schroeder, C., Gibson, L., Nordstrom, C. \& Beug, H. The estrogen receptor cooperates with the TGF alpha receptor (c-erbB) in regulation of chicken erythroid progenitor self-renewal. EMBO J 12, 951-960 (1993).

42 Hoegg, S., Brinkmann, H., Taylor, J. S. \& Meyer, A. Phylogenetic timing of the fish-specific genome duplication correlates with the diversification of teleost fish. J Mol Evol 59, 190-203, doi:10.1007/s00239004-2613-z (2004).

43 Amores, A., Catchen, J., Ferrara, A., Fontenot, Q. \& Postlethwait, J. H. Genome evolution and meiotic maps by massively parallel DNA sequencing: spotted gar, an outgroup for the teleost genome duplication. Genetics 188, 799-808, doi:10.1534/genetics.111.127324 (2011).

44 Nei, M. \& Roychoudhury, A. K. Probability of fixation and mean fixation time of an overdominant mutation. Genetics 74, 371-380 (1973). 
45 Takahata, N. \& Maruyama, T. Polymorphism and loss of duplicate gene expression: a theoretical study with application of tetraploid fish. Proceedings of the National Academy of Sciences of the United States of America 76, 4521-4525 (1979).

46 Watterson, G. A. On the time for gene silencing at duplicate Loci. Genetics 105, 745-766 (1983).

47 Force, A. et al. Preservation of duplicate genes by complementary, degenerative mutations. Genetics 151, 1531-1545 (1999).

48 Dolznig, H., Bartunek, P., Nasmyth, K., Mullner, E. W. \& Beug, H. Terminal differentiation of normal chicken erythroid progenitors: shortening of G1 correlates with loss of D-cyclin/cdk4 expression and altered cell size control. Cell Growth Differ 6, 1341-1352 (1995).

49 Chabot, B., Stephenson, D. A., Chapman, V. M., Besmer, P. \& Bernstein, A. The proto-oncogene ckit encoding a transmembrane tyrosine kinase receptor maps to the mouse W locus. Nature 335, 88-89, doi:10.1038/335088a0 (1988).

50 Svoboda, O. et al. Dissection of vertebrate hematopoiesis using zebrafish thrombopoietin. Blood 124, 220-228, doi:10.1182/blood-2014-03-564682 (2014).

51 Ransom, D. G. et al. Characterization of zebrafish mutants with defects in embryonic hematopoiesis. Development 123, 311-319 (1996). 


\section{Figure legends}

Figure 1. The effect of zebrafish Kitlga and Kitlgb on self-renewal and proliferation of hematopoietic cells ex vivo.

(A, B) Quantification of the number of whole kidney marrow cells after treatment with PBS (control) or specific cytokine combinations for 3 days (A) and 7 days (B). Statistical significance was assessed using standard one-way ANOVA. n.s. $=p>0.05, * p \leq 0.05, * * p \leq 0.01, * * * p \leq 0.001, * * * * p \leq 0.0001$.

(C) Growth curve for ex vivo culture of whole kidney marrow cells with different cytokine combinations. Biological duplicates for every condition and timepoint are shown. Representative photomicrographs (right) of whole kidney marrow cells isolated from transgenic gata1:DsRed fish treated with Epo, Dex and Kitlga and cultivated for 8 days. Images were acquired on Olympus IX70 inverted microscope equipped with the Olympus DP72 camera using a 20x objective. Scale bar represents 100 um.

(D, E) An expression heatmap of Epo, Dex and Epo, Dex, Kitlga treated cells showing the top predicted differentially expressed genes (D) and representative markers of erythroid differentiation (E). Color coding by z-score for heatmaps in $\mathrm{E}$ and $\mathrm{F}$.

\section{Figure 2. In vivo effects of kitlga and kitlgb at $72 \mathrm{hpf}$.}

(A) Representative micrographs of Icr:EGFP transgenic reporter larvae at $72 \mathrm{hpf}$ after injection of corresponding mRNAs. Control represents uninjected larvae. Images were acquired on Zeiss Axio Zoom.V16 with Zeiss Axiocam 506 mono camera and ZEN Blue software.

(B) Quantification of the Icr:EGFP positive cells in embryonic tails from fish in (A). Values were plotted using the mean of epo as the relative expression fold 1.

(C) FACS analysis of Icr:EGFP positive cells in whole embryos.

(D) Representative micrographs of benzidine stained embryonic tails at $72 \mathrm{hpf}$ after injection of corresponding mRNAs. Control represents uninjected embryos. NOTE increase in pigmentation in the dorsal part of kitlga-injected embryos. Z-stacks were acquired on Zeiss Axio Zoom.V16 with Zeiss Axiocam 105 color camera and processed using Extended Depth of Focus module in the ZEN blue software.

(E) Quantification of the benzidine stained embryonic tails in (D). Values were plotted using the mean of epo as the relative expression fold 1. 
(F) qPCR expression analysis of gata1a and hbbe1 from whole injected embryos at $72 \mathrm{hpf}$. Data have been normalized using mob4 as the housekeeping gene and using uninjected control as the relative expression fold 1. Bars represent a mean of three triplicates with SD. n.s. $=p>0.05, * p \leq 0.05, * * p \leq 0.01, * * * p \leq$ $0.001, * * * * p \leq 0.0001$.

Figure 3. Expression of kit receptors in the development.

(A) qPCR expression analysis of kita (left) and kitb (right) at different developmental stages. Data was normalized using ef $1 a$ as the housekeeping gene and using the $24 \mathrm{hpf}$ sample as relative expression fold 1.

(B) Sorting strategy used for FACS isolation of gata1:DsRed positive cells (top left) from transgenic zebrafish at $24 \mathrm{hpf}$ stage. qPCR expression analysis of gata1 (top right), kita (bottom left) and kitb (bottom right) in gata1:DsRed negative and positive cells. Data was normalized using ef1a as the housekeeping gene and using the gata1:DsRed positive $24 \mathrm{hpf}$ sample as relative expression fold 1.

(C) Sorting strategy used for FACS isolation of Icr:EGFP positive cells (top left) from transgenic zebrafish at $24 \mathrm{hpf}$ stage. qPCR expression analysis of gata1 (top right), kita (bottom left) and kitb (bottom right) in Icr:EGFP negative and positive cells. Data was normalized using ef1a as the housekeeping gene and using the Icr:EGFP positive $24 \mathrm{hpf}$ sample as relative expression fold 1.

\section{Figure 4. The effect of kitlga and kitlgb in sparse mutants.}

(A) Quantification of the benzidine staining in the tail region of control (uninjected) or epo, kitlga and kitlgb mRNA injected wild-type or kita mutant embryos (sparse). Values were plotted relatively to the mean of epo (=1). NOTE that for demonstrative purposes, Figure 4A left is re-used from Figure 2C.

(B) qPCR expression analysis of gata1a and hbbe1 from whole injected kita mutant embryos (sparse) at $72 \mathrm{hpf}$. Data was normalized using mob4 as the housekeeping gene and uninjected control as the relative expression fold 1. Bars represent mean of three triplicates with SD. n.s. $=p>0.05, * p \leq 0.05, * * p$ $\leq 0.01, * * * \mathrm{p} \leq 0.001, * * * * \mathrm{p} \leq 0.0001$. 


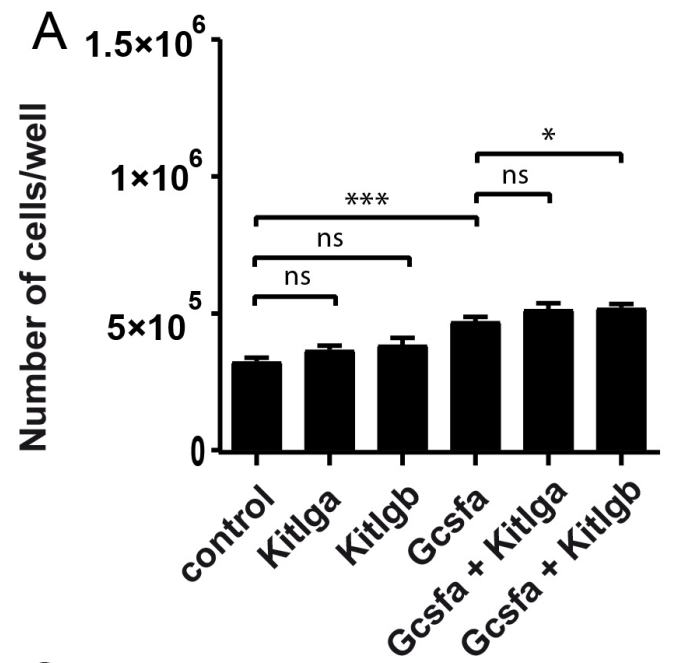

C

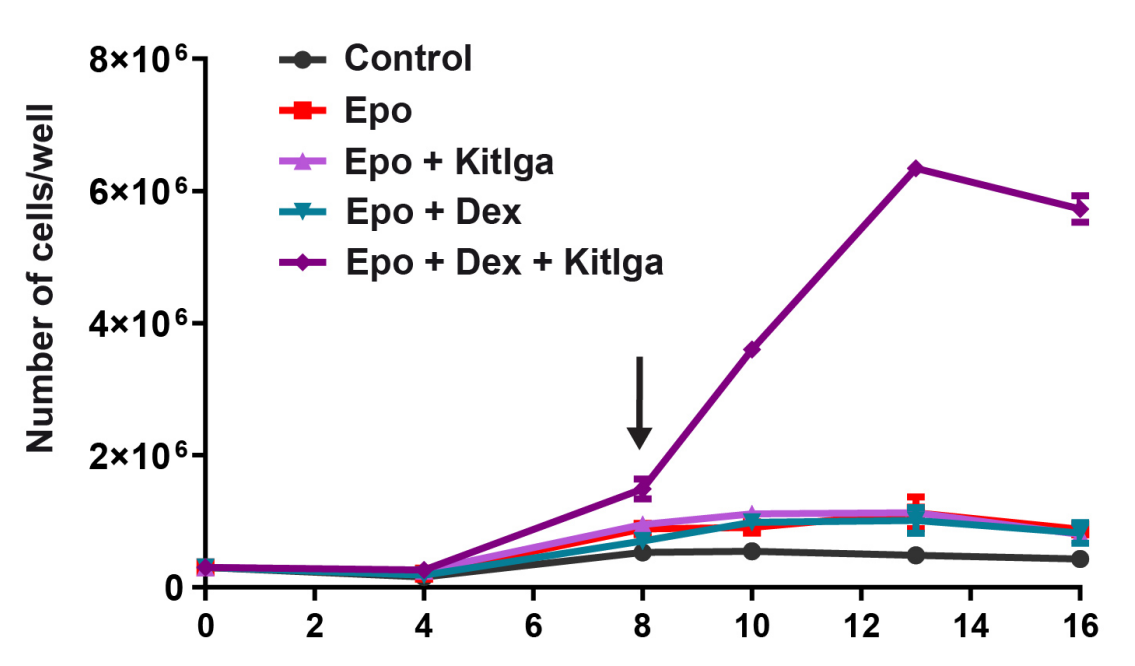

Days in culture
B

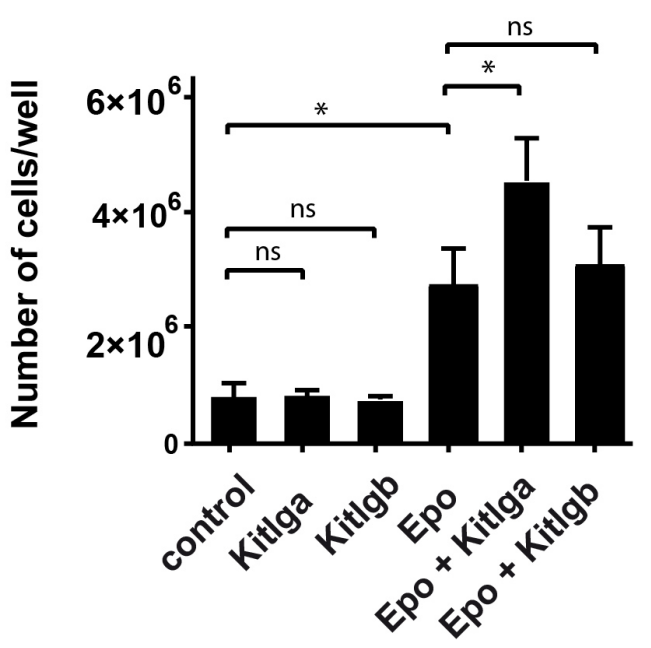

$\mathrm{E}$
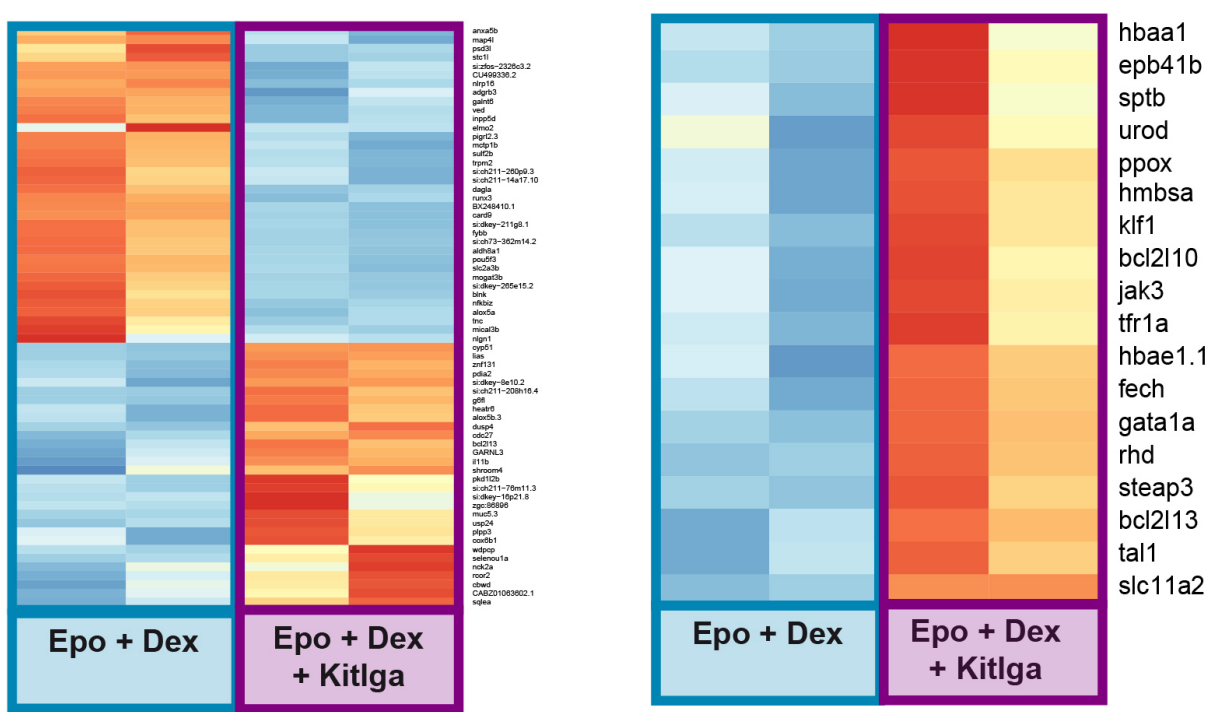

color key

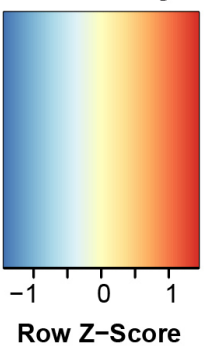

Row Z-Score

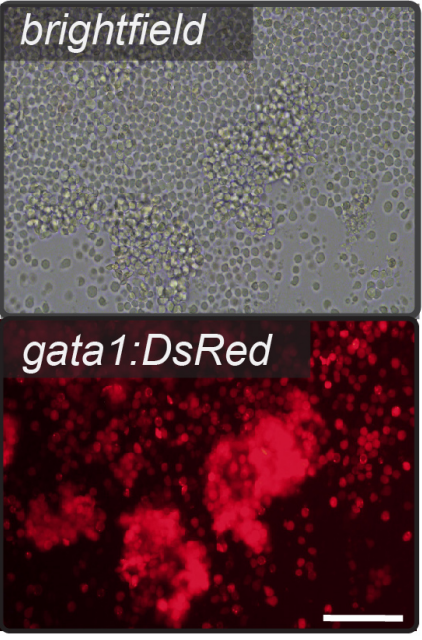



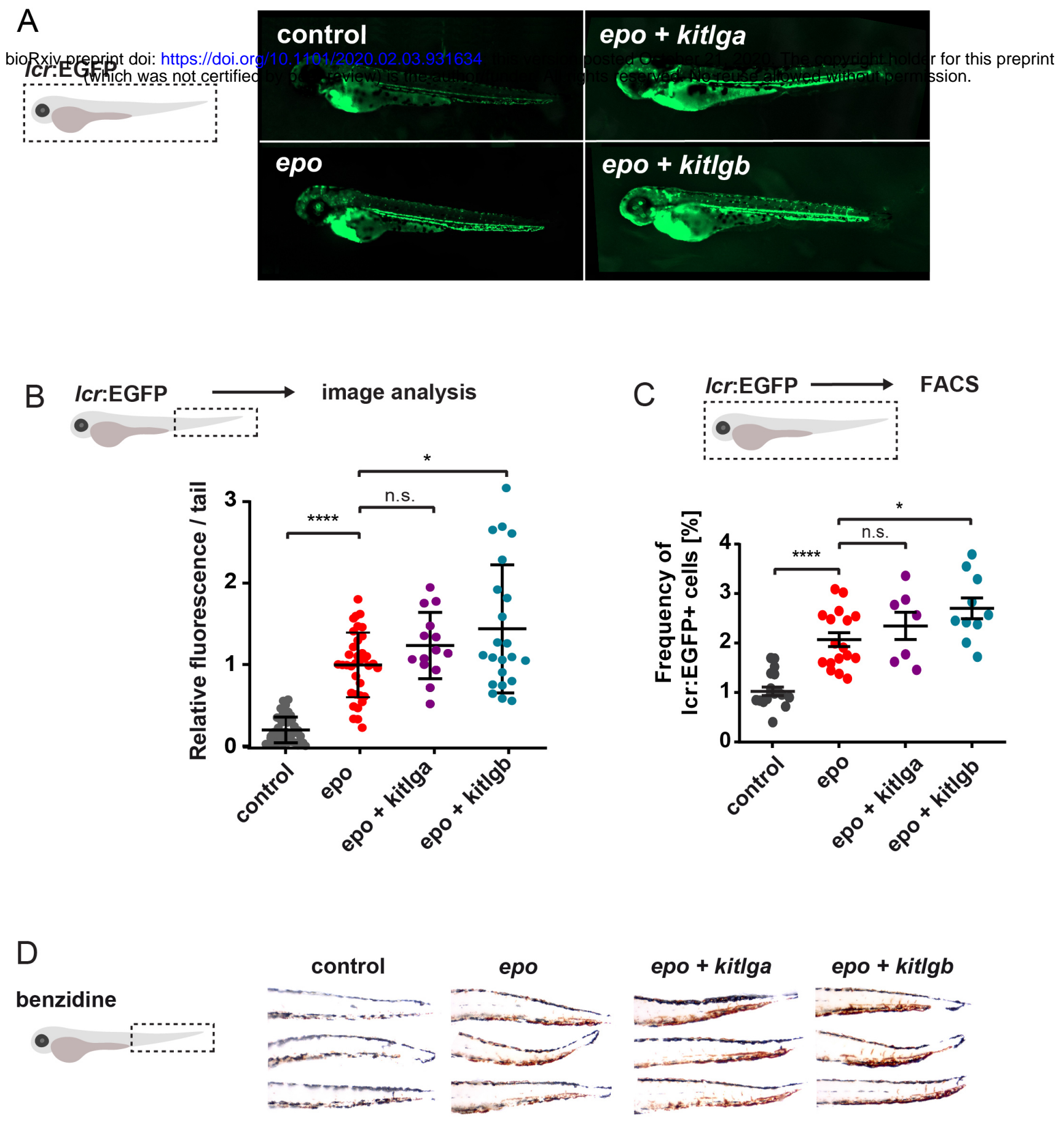

E
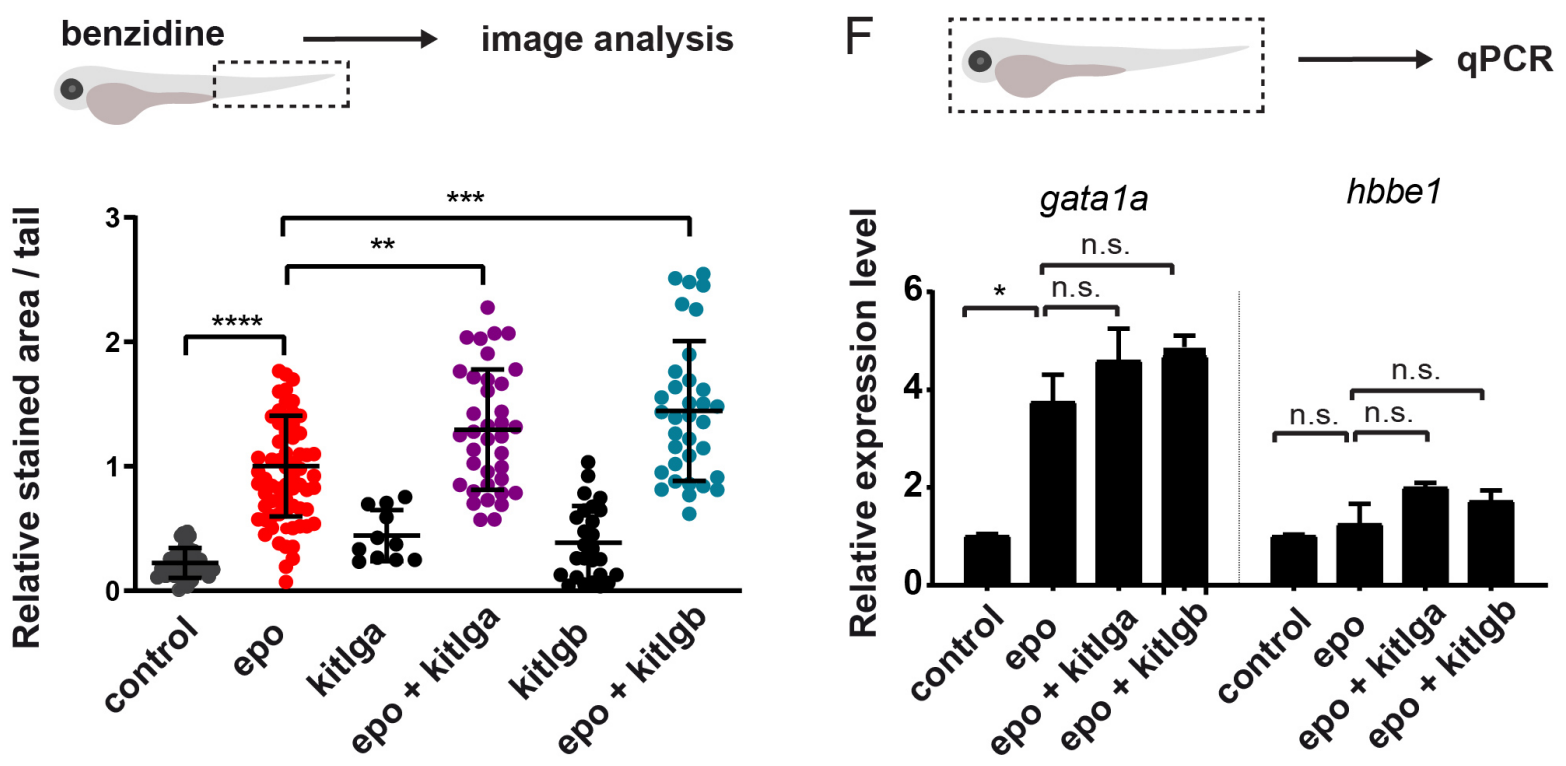
bioRxiv preprint doi: https://doi.org/10.1101/2020.02.03.931634; this version posted October 21, 2020. The copyright holder for this preprint (which was not certified by peer review) is the author/funder. All rights reserved. No reuse allowed without permission.

$$
\text { A }
$$
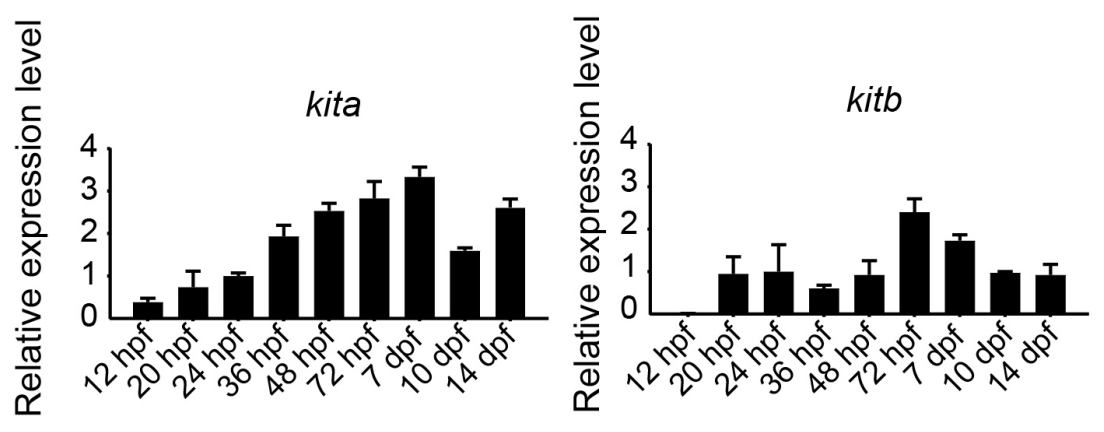

B
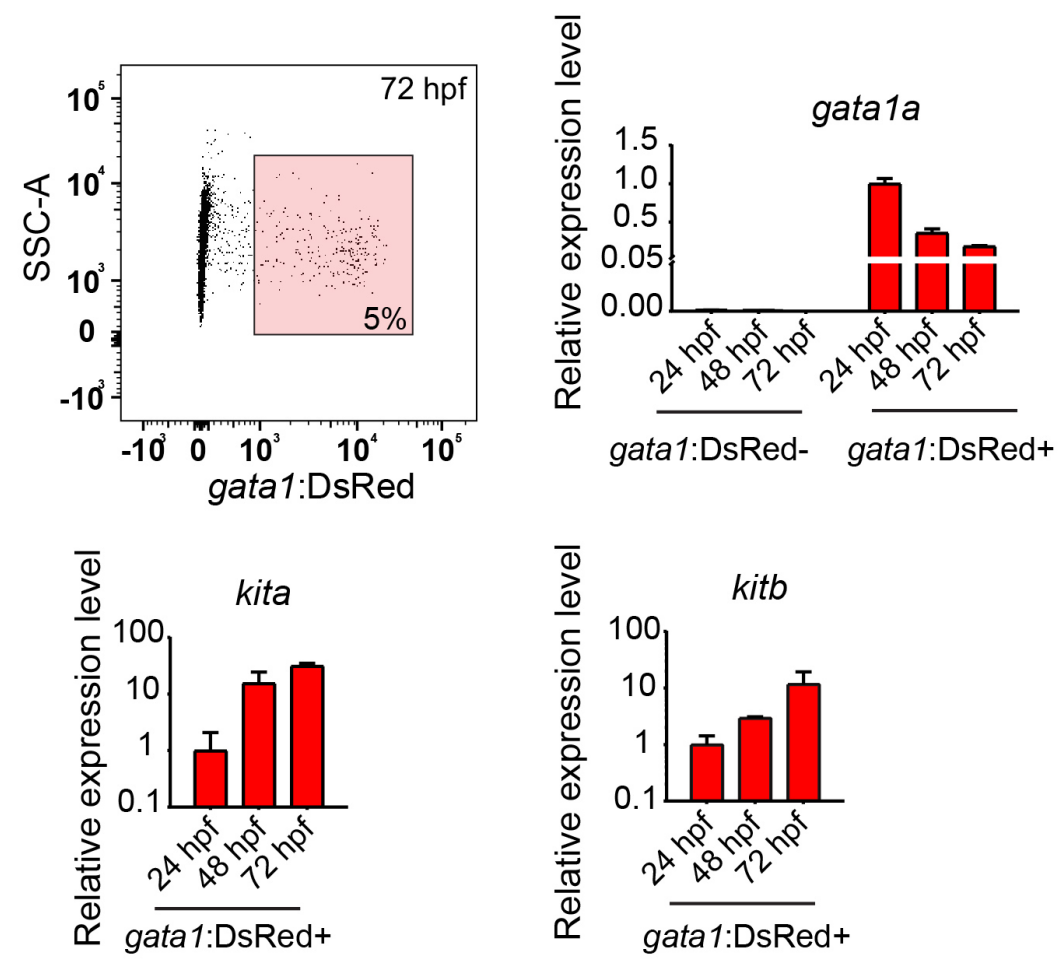

C
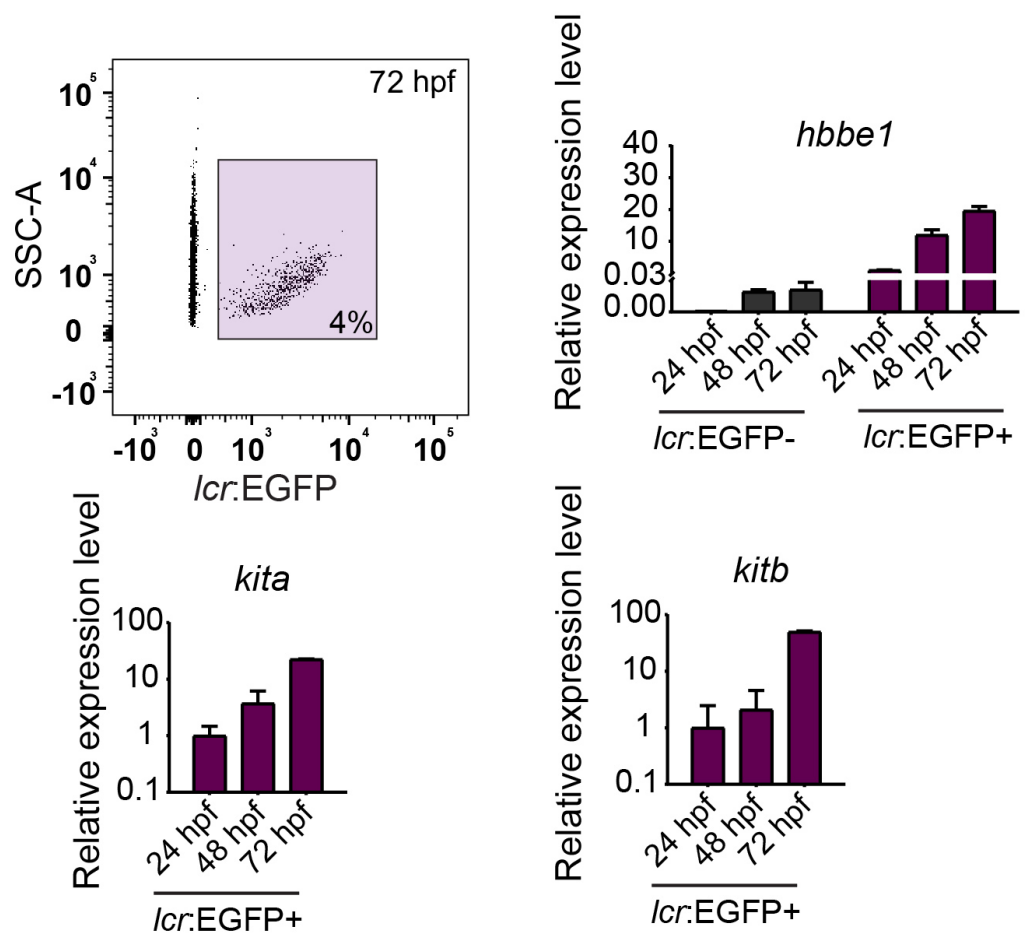

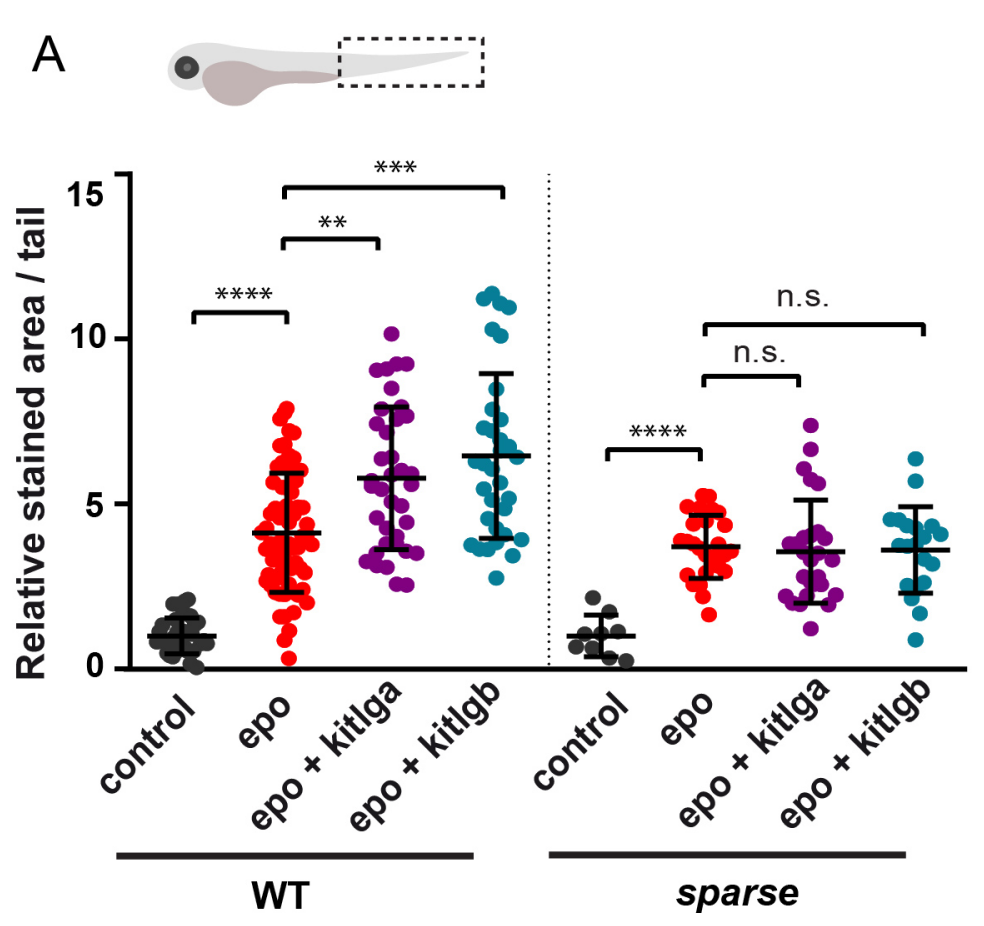
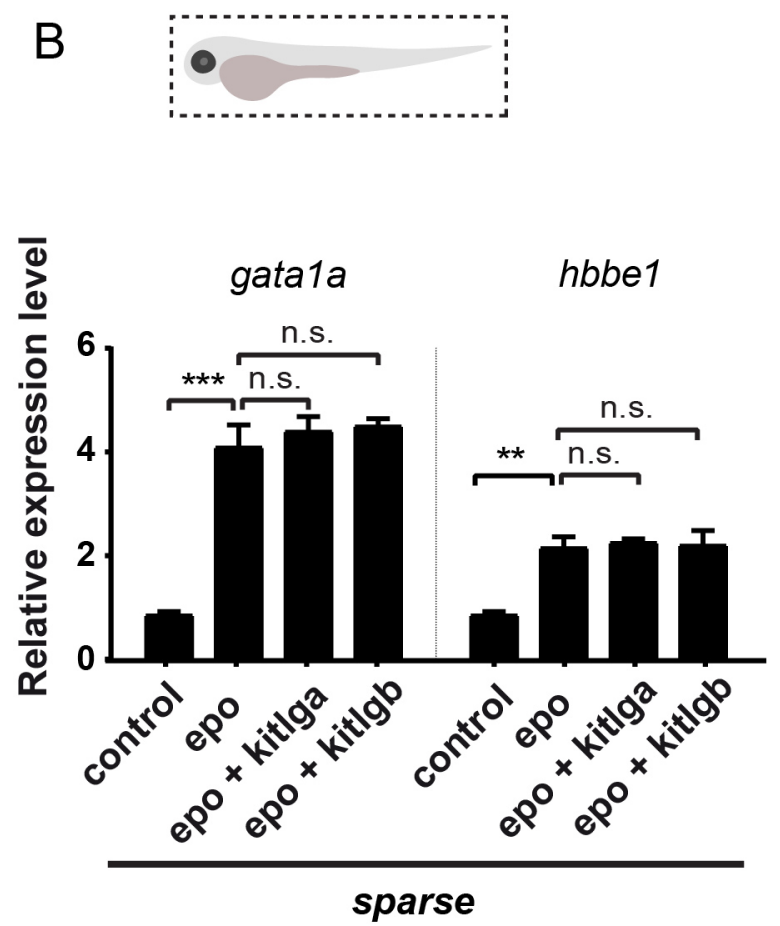